Audiology

Neurotology
Audiol Neurotol 2012;17:54-68

DOI: $\underline{10.1159 / 000329364}$
Received: October 13, 2010

Accepted after revision: April 11, 2011

Published online: August 9, 2011

\title{
Interaural Stacked Auditory Brainstem Response Measures for Detecting Small Unilateral Acoustic Tumors
}

\author{
Manuel Don ${ }^{\mathrm{a}}$ Betty Kwong ${ }^{\mathrm{b}}$ Chiemi Tanaka ${ }^{\mathrm{c}}$ \\ aElectrophysiology Laboratory, Division of Communication and Auditory Neuroscience, House Research Institute, \\ Los Angeles, Calif., ${ }^{b}$ Former member and current consultant to the Electrophysiology Laboratory, and \\ 'Oregon Hearing Research Center, Department of Otolaryngology, Oregon Health and Science University, \\ Portland, Oreg., USA
}

\section{Key Words}

Interaural Stacked auditory brainstem responses $\cdot$ Acoustic tumor $\cdot$ Vestibular schwannoma $\cdot$ Derived-band auditory brainstem responses $\cdot$ High-pass masking

\begin{abstract}
The Stacked auditory brainstem response (SABR) was developed and investigated as a screening tool for small $(\leq 1 \mathrm{~cm})$ unilateral acoustic tumors (vestibular schwannomas) that were missed by standard clinical auditory brainstem response (ABR) measures [Don et al.: Am J Otol 1997;18:608621; Audiol Neurotol 2005;10:274-290]. While the SABR measure provided much greater sensitivity than the standard $A B R$ measures for small tumor detection, we believed that the large intersubject variability of the SABR measure compromised both the sensitivity and specificity of the measure. However, as we demonstrate in this paper, the variability between ears of a given individual is small. Thus, we introduced an interaural SABR (ISABR) amplitude difference measure to improve the sensitivity and specificity of the SABR amplitude measure to detect small unilateral acoustic tumors. Its main advantages are two-fold. First, it is somewhat immune to variables that affect the absolute SABR amplitudes because it is a relative measure. Second, it is better at assessing tumor
\end{abstract}

patients with very large and non-tumor patients with very small absolute SABR amplitudes. We believe that the ISABR is a useful addition to $A B R$ measures aimed at detecting the presence of unilateral acoustic tumors.

Copyright $\odot 2011$ S. Karger AG, Basel

\section{Introduction}

We developed and investigated the Stacked auditory brainstem response (SABR) as a screening tool for small $(\leq 1 \mathrm{~cm})$ unilateral acoustic tumors (vestibular schwannomas) that were missed by standard clinical auditory brainstem response (ABR) measures [Don et al., 1997, 2005; review by Musiek et al., 2007]. Our hypothesis [Don et al., 1997, 2005; Don and Kwong, 2009] is that the failure of the standard clinical ABR measures to detect small tumors is due to their reliance on latency changes of wave $\mathrm{V}$ of the ABR and that (1) high-frequency fibers dominate the standard click-evoked ABR latency measure, (2) tumors will be missed if these high-frequency fibers are not sufficiently affected by the tumor, and (3) small tumors do not always affect the subset of high-frequency fibers sufficiently. In these studies, we also hypothesized that a solution to the problem of detecting small tumors is to

\section{KARGER}

(c) 2011 S. Karger AG, Basel

Fax +4161306 1234 E-Mail karger@karger.ch www.karger.com www.karger.com/aud
Manuel Don, $\mathrm{PhD}$

Electrophysiology Laboratory, Division of Communication and Auditory Neuroscience, House Research Institute, 2100 W. Third Street, Fifth Floor Los Angeles, CA 90057 (USA)

Tel. +1 213353 7095, E-Mail mdon@ hei.org 
use a measure that assesses activity from essentially all nerve fibers, not just a subset. The proposed solution, the SABR, is related to the total amount of synchronous neural activity evoked by click stimulation. Using the derived-band $A B R$ and stacking techniques, the SABR is formed by temporally aligning and summing the synchronous activity of octave-wide bands of activity initiated across the cochlea in response to click stimulation [Don et al., 1994, 1997, 2005]. Because the SABR is composed of neural activity initiated across the whole cochlea, reduction of activity by a small tumor will reduce the SABR amplitude. These papers demonstrated that the SABR amplitude measure can be a highly sensitive, widely available, cost-effective, and comfortable tool for screening small acoustic tumors (SAT). The SABR measure can be of even greater value when screening with magnetic resonance imaging (MRI) is not feasible, wanted or available. In the papers cited, the criterion SABR amplitude that provided 95\% sensitivity (correct detection of small tumor patients) was referenced to the right ear SABR amplitudes of non-tumor normal-hearing (NTNH) subjects. The right ear was arbitrarily chosen as the reference because it was assumed that the SABR amplitudes would be similar for the two ears. As shown later in this paper (fig. 6), the assumption seems warranted. Thus, for the single ear comparison in Don et al. [2005], it did not matter which ear of the NTNH subjects was used as the reference. The $95 \%$ sensitivity criterion resulted in $88 \%$ specificity (correct identification of nontumor subjects). The SABR is particularly targeted at individuals with small tumors who have normal standard ABR measures (e.g. interaural wave V difference or IT5) and minimal hearing loss. Because the standard IT5 is a relatively fast and simple test that detects nearly all medium and large tumors and $50-70 \%$ of small tumors [Don, 2002], it should be the first clinical test [Don and Kwong, 2009]. The SABR should be performed only when the IT5 appears normal and the clinician feels that retrocochlear involvement is still a reasonable possibility [Don and Kwong, 2009].

The basic principle of the SABR method is that a small tumor should reduce the SABR amplitude to a level below criterion. In the study by Don et al. [2005], the mean reduction observed for the 54 SAT patients relative to the $78 \mathrm{NTNH}$ subjects was approximately $50 \%$. That is, by the time the patient seeks medical attention for his/her symptoms, synchronous neural activity has been reduced, on average, by $50 \%$. Even with this average amount of reduction, it is conceivable that in individuals with normally very large SABR amplitudes, a tumor may still not reduce the SABR amplitude below the criterion level. For example, if an individual who would normally have a SABR that is twice as large as the mean for NTNH subjects had a small tumor that reduced the SABR by $50 \%$, the SABR amplitude would then be equivalent to the mean of NTNH subjects and fall within the normal criterion limits. Thus, the tumor would be missed and the test's sensitivity would be compromised. While the percentage of individuals having SABRs that are twice the size of the mean value is small, it is a potential problem. In addition, if the average reduction is 50\%, there will obviously be a number of cases where the reduction is less than as well as greater than $50 \%$. Those tumors that reduce the SABR amplitude by less than $50 \%$ may be missed as well. This problem of missing tumors in individuals with normally very large SABRs or reductions of less than $50 \%$ can be minimized if it can be shown that the SABR is typically very similar between ears such that an interaural comparison can be used, much like the standard IT5 latency measure developed by Selters and Brackmann [1977]. Likewise, specificity could be improved for individuals who would normally have low SABR amplitudes or amplitudes that are low due to either hearing loss or age [Don et al., 2005]. In these individuals, with the exception of bilateral tumor cases, equal but below criterion SABR amplitudes in both ears would be suggestive of a non-tumor subject. As we will discuss later, interaural SABR (ISABR) comparisons are not always warranted or feasible so single ear data [Don et al., 2005] must be used in some cases. However, when feasible and valid, the interaural measure may be helpful because it uses the individual patient as his/her own control rather than absolute values of the SABR amplitude generated by a specific population. In this paper, we report on an ISABR amplitude measure in NTNH subjects and patients with SATs.

\section{Methods}

This investigation received Institute Review Board approval. The purpose and procedure of the study were orally presented by the experimenter. The experimenter then answered any questions the subject had regarding his/her participation. Finally, each subject read and signed an Institute Review Board approved informed consent form.

Subjects

NTNH Control Group

A control group of 39 NTNH subjects in good general health and reported normal neurological and otologic status was tested. The NTNH subjects were recruited from (1) staff members of the House Research Institute and House Clinic, (2) friends and rela- 
Table 1. Characteristics of NTNH subjects $(n=39)$

\begin{tabular}{llll}
\hline & Males & Females & Total \\
\hline Number of subjects & 19 & 20 & 39 \\
Age range, years & $18-39$ & $19-39$ & \\
Mean age, years & 27.3 & 29.4 & \\
\hline
\end{tabular}

The NTNH subjects were recruited from House Research Institute and House Clinic employees, their families and friends, House Clinic patients with normal MRI results and students at local colleges.

Table 2. Characteristics of SAT patients $(n=17)$

\begin{tabular}{lccc}
\hline & Males & Females & Total \\
\hline Right-ear tumor & 3 & 2 & 5 \\
Left-ear tumor & 6 & 6 & 12 \\
Number of patients & 9 & 8 & 17 \\
Age range, years & $38-66$ & $36-62$ & \\
Mean age, years & 50.8 & 48.9 & \\
\hline
\end{tabular}

Patients were classified as having SATs if they had small $(\leq 1 \mathrm{~cm})$ tumors, irrespective of standard ABR results or tumors undetected by standard ABR measures, irrespective of tumor size.

tives of the staff members, (3) patients with negative MRI results, and (4) students at local colleges. Otoscopic examinations were performed to identify existing conditions that would preclude audiometric and ABR testing. At the time of ABR data collection, pure-tone audiometric testing was performed with a GrasonStadler GSI 61 audiometer and ER-3A insert earphones. Hearing thresholds were evaluated in $5 \mathrm{~dB}$ steps using the modified Hughson-Westlake procedure [Carhart and Jerger, 1959]. Normal hearing was defined as pure-tone thresholds (PTT) of $10 \mathrm{~dB}$ HL or less for the 7 frequencies tested between and including 500 and 4000 $\mathrm{Hz}(500,750,1000,1500,2000,3000,4000 \mathrm{~Hz}) ; 15 \mathrm{~dB}$ HL or less for 6000 and $8000 \mathrm{~Hz}$. Gender and age of the subjects of the NTNH control group are presented in table 1.

\section{SAT Patients}

The test population for this study was restricted to patients recruited from the House Clinic who met the following three criteria: (1) clinical complaints related to hearing or balance, (2) a Gd-DTPA enhanced MRI indicating the presence of a tumor, and (3) a tumor that was $\leq 1.0 \mathrm{~cm}$, irrespective of the $\mathrm{IT} 5$ results, or undetected by the standard IT5 ABR measure, irrespective of its size. The first restriction is important because individuals without symptoms normally would not be clinically evaluated. The major exceptions are patients at risk for neurofibromatosis type II. Neurofibromatosis type II patients without clinical signs were excluded from the study. A total of 17 small tumor patients were tested in both ears. Gender, age, and tumor ear of the subjects of the SAT test group are presented in table 2 . The tumor was surgically confirmed in all cases except for two patients who elected not to have surgery. The diagnoses of acoustic tumors were based on the MRI evaluations.

Stimuli

The stimuli were rarefaction clicks produced by applying to an ER-2 insert phone $100 \mu \mathrm{s}$ voltage pulses that were generated, filtered, and amplified by an Ariel DSP-16 board. The clicks were presented $22 \mathrm{~ms}$ apart ( $\sim 45$ clicks/s) at $60 \mathrm{~dB}$ above the average perceptual detection threshold for a group of 19 normal-hearing subjects (i.e. $60 \mathrm{~dB} \mathrm{nHL}$ ).

Ipsilateral pink-noise masking was used to obtain the derivedband ABRs [Don and Eggermont, 1978] that are required to form the SABR. The RMS level of the broadband pink noise produced by a General Radio Noise Generator (Type 1310) was $81 \mathrm{~dB}$ SPL. This level represents the average of the broadband pink noise levels needed to mask $60 \mathrm{~dB}$ nHL click-evoked ABRs in the group of 19 normal-hearing subjects tested for perceptual detection thresholds. There were six stimulus conditions: clicks presented alone and clicks presented with ipsilateral noise high-pass filtered at 8 , 4, 2, 1, and $0.5 \mathrm{kHz}$. The slope of the high-pass filtered masking noise was $96 \mathrm{~dB} /$ octave and was achieved by cascading both channels of a Krohn-Hite (Model 3343) dual filter, each with a 48-dB/ octave slope.

\section{ABR Recordings}

Subjects were placed in a reclining chair in a double-walled sound-treated room (IAC). ABRs were recorded differentially between electrodes applied to the vertex $(\mathrm{Cz})$ and the ipsilateral mastoid (M1 or M2). The electrode on the contralateral mastoid was used as ground. Scalp activity was amplified by $5 \times 10^{5}$ and filtered (cascading two Princeton Applied Research Model 113) with a passband of 0.1 to $3 \mathrm{kHz}$ (slope $=12 \mathrm{~dB}$ /octave). The activity was sampled at a rate of $20 \mathrm{kHz}$ for $15 \mathrm{~ms}$ after stimulus onset with use of an Ariel DSP-16 A/D-D/A board and a PC. After each block of 256 sweeps, the RMS value of the averaged background noise was estimated according to procedures of Elberling and Don [1984]. This noise estimate was used to form a weighted average by applying Bayesian estimation principles [Elberling and Wahlgreen, 1985; Don and Elberling, 1994]. This technique reduces the destructive effects of episodic physiological background noise variation on the ABR average by weighting the average towards those blocks of sweeps with low estimated background noise. Data collection for a run was terminated when the estimated residual background noise in the average reached $20 \mathrm{nV}$ RMS or less. Thus, all the recordings had approximately the same low residual background noise levels. Using a low residual background noise level as the stopping criterion rather than a fixed number of sweeps reduces variations in the averaged physiological background noise that can have a substantial effect on the interpretation of the ABR recordings [Don and Elberling, 1994, 1996]. In addition, by making sure that the residual noise in all our ABR averages is low, we can be confident that the responses we measure represent mostly neural activity and not unaveraged physiological noise. This low residual noise requirement is particularly important for the SABR measure. 


\section{ABR Data Measurements and Analyses}

Interaural Wave V Latency Difference (IT5)

The IT5 [Selters and Brackmann, 1977] is a normalized measure that compares the difference in wave $\mathrm{V}$ latencies between ears of the same subject. In unilateral tumor patients, the wave $\mathrm{V}$ latency from the tumor ear is expected to be significantly longer than the wave $\mathrm{V}$ latency in the non-tumor ear. According to the criterion recommended by Selters and Brackmann [1977], if the difference is greater than $0.2 \mathrm{~ms}$ after compensating for hearing loss at $4 \mathrm{kHz}$, the test result is positive for a tumor. The correction factor of $0.1 \mathrm{~ms}$ for every $10 \mathrm{~dB}$ of hearing loss above $50 \mathrm{~dB}$ at $4 \mathrm{kHz}$ used by Selters and Brackmann [1977] was derived empirically and graphically rather than determined statistically (see fig. 5). Subsequent studies by Rosenhamer and his associates examined the effect of cochlear hearing loss on the latency of wave $\mathrm{V}$ of the ABR and found that for their subject populations, it was best if the correction began at losses above $30 \mathrm{~dB} H \mathrm{HL}$ at $4 \mathrm{kHz}$ [Rosenhamer et al., 1981] instead of the $50 \mathrm{~dB}$ described by Selters and Brackmann [1977].

As mentioned earlier, the standard IT5 ABR measure [Selters and Brackmann, 1977] is recommended as the first test to perform [Don and Kwong, 2009]. Although it misses 30-50\% of small tumor cases, it does detect nearly all medium and large tumors [Don, 2002] and thus, the majority of tumor cases. If the IT5 test is normal and a tumor is suspected, we recommended that the SABR test then be applied [Don and Kwong, 2009].

For both the NTNH and SAT groups, an IT5 measure was obtained to $60 \mathrm{~dB}$ nHL clicks. For the SAT patients, the wave V latency from the non-tumor ear was subtracted from the wave $\mathrm{V}$ latency of the tumor ear. Negative IT5 values indicate that the wave $\mathrm{V}$ latency of the tumor ear was shorter than that of the nontumor ear. For the NTNH subjects, there was no tumor ear, so the IT5 was calculated in two ways: (1) for the NTNH (R) data, the left ear latency was subtracted from that of the right ear, and (2) for the NTNH (L) data, the right ear latency was subtracted from that of the left ear. Thus, for the NTNH (R) condition, negative IT5 values indicate that the wave $\mathrm{V}$ latency from the right ear response was shorter than that from the left ear. Similarly, for the NTNH (L) condition, negative IT5 values indicate that the wave V latency was shorter on the left than the right.

SABR Amplitude

The derived-band ABRs and their use in constructing the SABR have been presented in detail elsewhere [Don et al., 1994, $1997,2005]$. The SABR is obtained by (1) temporally aligning the derived-band $A B R$ waveforms so that the peak latencies of wave $\mathrm{V}$ in each derived band coincide, and (2) adding together these aligned derived-band ABR waveforms. By temporally aligning the peak activity initiated from each segment of the cochlea, we synchronize the total activity and remove the phase-canceling effect that occurs in the standard response. The SABR amplitude is the peak-to-trough measure of wave V in the SABR. Thus, compared to standard ABR amplitude measures, the amplitude of the SABR wave $V$ reflects more directly the total amount of activity initiated across the cochlea. If activity initiated from any part of the cochlea is blocked or desynchronized by a tumor at the 8 th nerve level, then the SABR amplitude will be reduced.

The Kolmogorov-Smirnov test of normality was performed on the Stacked and standard ABR measures investigated in this study. The results indicated that none of the data distributions

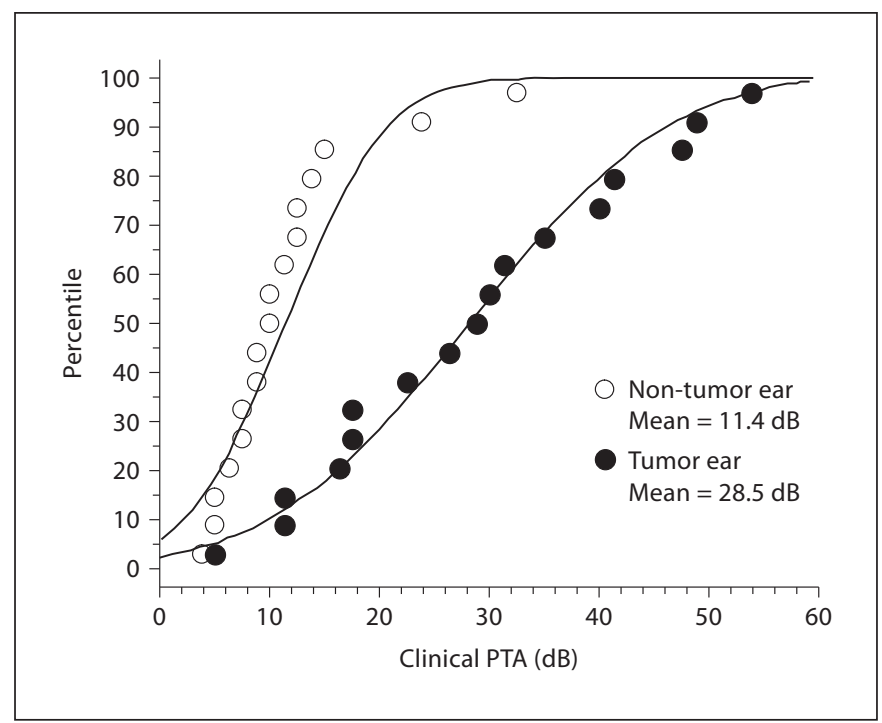

Fig. 1. Cumulative distributions for the clinical pure-tone averages (PTAs) for the non-tumor and tumor ears of the SAT group.

could be significantly distinguished from a Gaussian distribution described by the observed mean and standard deviation. Thus, it can be assumed that all the data reported here are normally distributed, justifying the use of normal parametric statistics in the analyses. One of the major analyses involves plotting the data in the form of cumulative normal distributions. These plots make it easy to quickly estimate the approximate sensitivity and specificity of the test measures. However, to maximize precision, especially when many values fall on or near the criterion value, the sensitivity and specificity numbers given in the text were calculated using the corresponding z-values of the Gaussian distributions, not simply estimated based on the cumulative distribution curves in the figures.

\section{Results}

\section{Audiometric Data}

Figure 1 plots the cumulative distributions for the clinical pure-tone average (PTA) for the non-tumor (open circles) and tumor ears (filled circles) of the SAT group. The clinical PTA is the average of the thresholds for the following four audiometric frequencies: $0.5,1,2$, and $3 \mathrm{kHz}$. The mean clinical PTAs were $11.4 \mathrm{~dB}$ and $28.5 \mathrm{~dB}$ for the non-tumor and tumor ears respectively. Thus, there was a clinical PTA mean difference of about $17 \mathrm{~dB}$ between the two ears.

In figure $2 \mathrm{a}$, for each of the 17 patients of the SAT population, the interaural difference in the clinical PTA (PTA in tumor ear - PTA in non-tumor ear) is plotted 


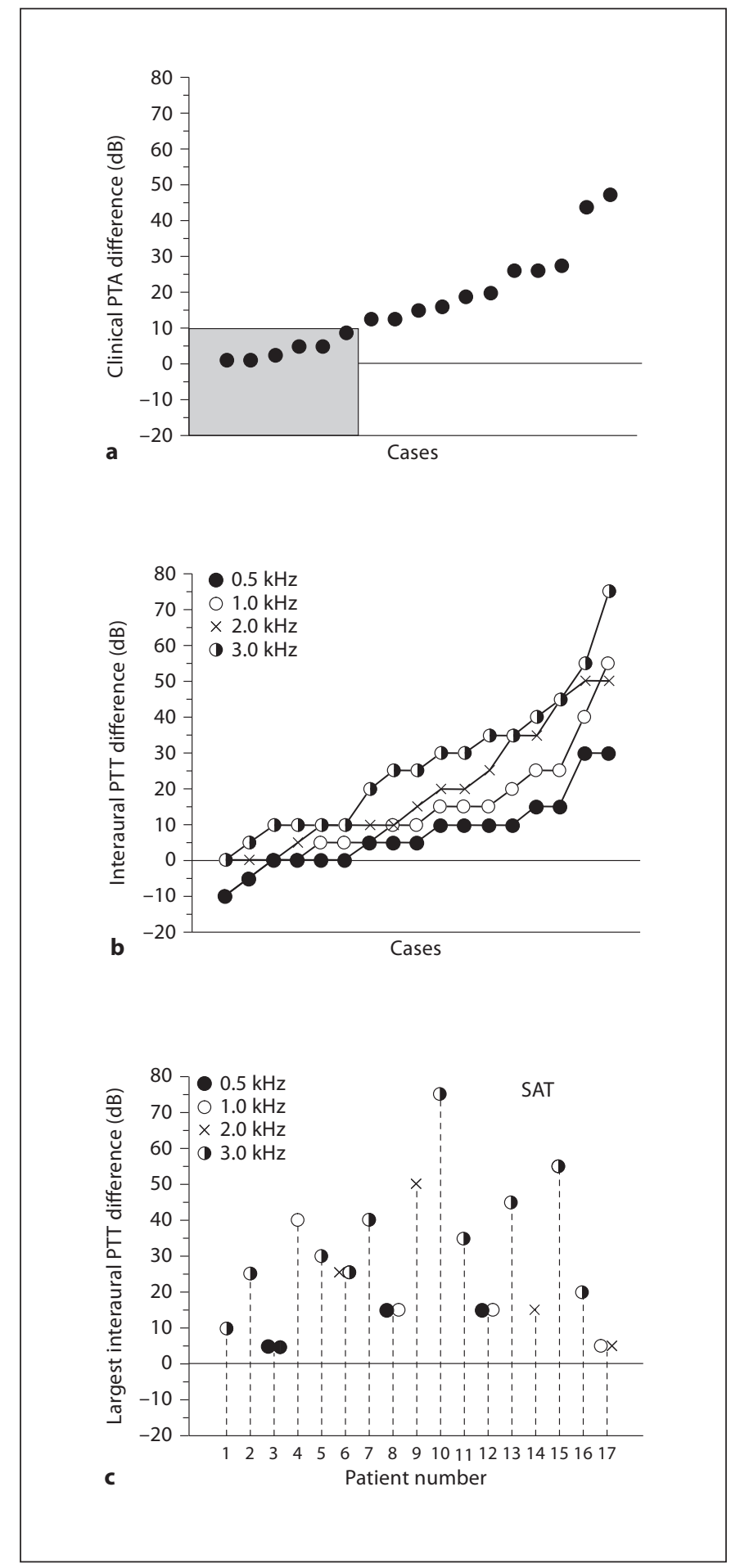

Fig. 2. For the SAT population, interaural differences are plotted in order, from the smallest to the largest for (a) the clinical puretone average (PTA) and (b) the individual pure-tone thresholds (PTTs) comprising the clinical PTA. In c, the tones having the largest interaural differences in PTT are plotted for each patient (see text for description of these results). and ordered from the smallest to the largest. Hearing loss due to the presence of a small tumor can be minimal so it is not surprising that in 6 patients (35\%), the interaural PTA difference is $10 \mathrm{~dB}$ or less (shaded area). In figure $2 \mathrm{~b}$, the interaural PTT differences (threshold in tumor ear - threshold in non-tumor ear) are plotted for each of the four frequencies used in calculating the clinical PTA. Overall, one can see that the interaural PTT differences are, for most patients, greater for the higher frequencies ( $\mathrm{X}$ and half-filled circle symbols for 2 and $3 \mathrm{kHz}$, respectively). However, it can also be seen that the interaural PTT difference for the highest frequency ( $3 \mathrm{kHz}$, half-filled circles) is $10 \mathrm{~dB}$ or less in 6 cases (35\%). Not surprisingly, 4 of these 6 cases were in the group of the 6 lowest clinical PTAs shown in figure 2a. In figure $2 c$, the frequency or frequencies having the largest PTT difference are shown for each of the 17 SAT patients. The interaural PTT difference for $3 \mathrm{kHz}$ was the largest for 9 patients and tied for the largest in 2 others (patients No. 3 and 6). For 5 patients (No. 3, 4, 8, 12 and 17), the largest interaural PTT difference was for the 0.5- and/or $1-\mathrm{kHz}$ tones. Thus, a small tumor clearly does not always have its greatest effect on the highest frequency in the clinical PTA since the interaural PTT difference was largest for the $3-\mathrm{kHz}$ tone in only about $65 \%$ of the patients.

\section{ABR Data}

Interaural Wave V Latency Difference (IT5)

The cumulative distributions of the interaural wave $\mathrm{V}$ latency difference (IT5) are plotted in figure 3 for both the NTNH and SAT groups. For the SAT group, the latency from the non-tumor ear was subtracted from the latency in the tumor ear. The IT5 was corrected for hearing loss at $4 \mathrm{kHz}$ as recommended by Selters and Brackmann [1977]. The current standard criterion of $0.2 \mathrm{~ms}$ recommended by Selters and Brackmann [1977] is shown by the vertical dotted line. The values in the shaded area $(\leq 0.2$ $\mathrm{ms}$ ) are considered within normal limits. Two tumor cases had borderline normal IT5 values of $0.2 \mathrm{~ms}$. If we interpret these two cases to be normal, then five tumor cases would have been missed by the IT5 measure. Based on the fitted cumulative distribution curve for the SAT population, the calculated sensitivity for the IT5 criterion value of $0.2 \mathrm{~ms}$ recommended by Selters and Brackmann [1977] is $78 \%$, which is similar to the visually estimated value shown in figure 3 .

As discussed earlier, for the NTNH group, there is no tumor ear. So first we subtracted the left ear latency from the right ear latency $(\mathrm{NTNH}(\mathrm{R})$; denoted by filled cir- 
Fig. 3. Cumulative distributions of the IT5 results for the NTNH and SAT groups. NTNH group: NTNH (R) = left ear latency subtracted from right ear latency; NTNH $(\mathrm{L})=$ right ear latency subtracted from left ear latency. See text for details.

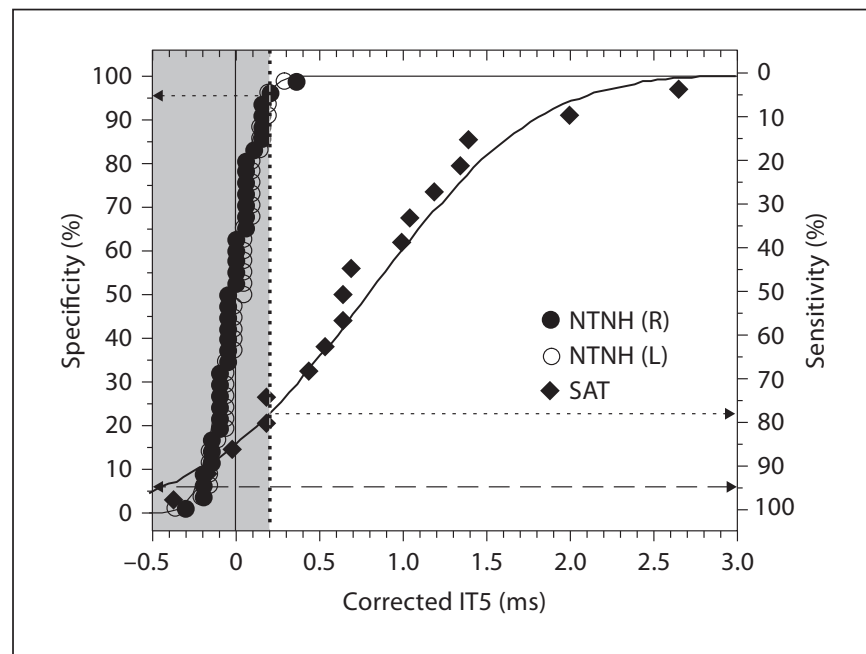

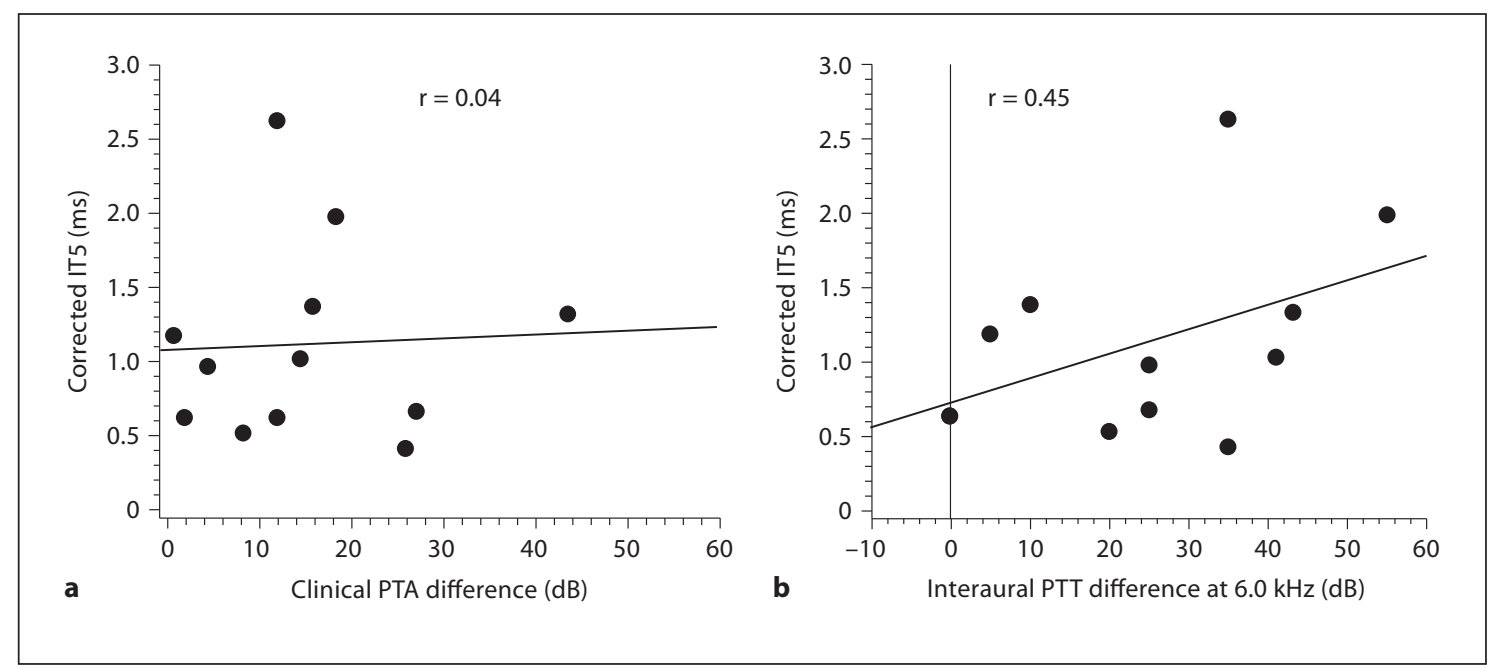

Fig. 4. For the 12 tumors detected by the IT5, the corrected IT5 value as a function of (a) the clinical PTA and (b) the interaural difference in pure-tone threshold (PTT) for $6 \mathrm{kHz}$ is displayed. In $\mathbf{b}$, only 11 instead of 12 separate points appear because two individuals have the same interaural difference at $6 \mathrm{kHz}(0 \mathrm{~dB})$ and the same IT5 value $(0.6 \mathrm{~ms})$.

cles), then the right ear latency from the left ear latency (NTNH (L); denoted by open circles). The fitted cumulative distribution curve in figure 3 illustrates the NTNH (R) condition. Based on these data, the calculated specificity for a criterion IT5 value of $0.2 \mathrm{~ms}$ is $95.6 \%$. For the NTNH (L) data, the calculated specificity is $99.9 \%$. Thus, the IT5 criterion of $0.2 \mathrm{~ms}$ recommended by Selters and Brackmann [1977] will yield about 97-98\% specificity on average. However, to insure that essentially all SAT patients are referred for imaging, the sensitivity (correct de- tection of small tumors) of the test must be considered more important than a high specificity (correct identification of non-tumor patients). If we use a $95 \%$ sensitivity criterion, we can estimate from figure 3 that this criterion would result in a negative IT5 value and yield a very low $(<5 \%)$ specificity. In fact, when using the corresponding z-values of the Gaussian distributions, for $95 \%$ sensitivity, the calculated IT5 value yields a specificity of less than $1 \%$. 
As noted earlier, 5 of the 17 tumor cases had IT5 values within normal limits $(\leq 0.2 \mathrm{~ms})$ and were missed by this measure. For the 12 tumors detected by the IT5, we wanted to determine if the detection might be related to various interaural behavioral measures. In figure 4 , we first plot the IT5 against the interaural clinical PTA difference. This plot, shown in figure $4 \mathrm{a}$, and the correlation analysis $(\mathrm{r}=0.04 ; \mathrm{p}=0.82)$, demonstrate that for these 12 tumor cases, the IT5 value is not related to the interaural clinical PTA difference. Similar analyses for each of the four frequencies that comprise the clinical PTA revealed no relationship between the frequencies and the tumor cases detected by the standard IT5. The correlation $\mathrm{r}$-values were $0.28(\mathrm{p}=0.37), 0.22(\mathrm{p}=0.49), 0.10(\mathrm{p}=0.76)$, and $0.06(\mathrm{p}=0.84)$ for $0.5,1.0,2.0$, and $3.0 \mathrm{kHz}$, respectively. However, note that the clinical PTA is composed of mid $(2$ and $3 \mathrm{kHz})$ and lower $(0.5$ and $1 \mathrm{kHz})$ frequencies. As discussed earlier, we have postulated [Don et al., 1997, 2005; Don and Kwong, 2009] that the IT5 measure relies on the tumor affecting a sufficient number of high-frequency fibers in order to cause a significant latency delay or shift in wave $\mathrm{V}$ of the ABR. Thus, there might be a relationship between detection with the IT5 and the interaural PTT difference in high frequencies. The plot and the correlation analysis $(\mathrm{r}=0.45 ; \mathrm{p}=0.14)$ in figure $4 \mathrm{~b}$ show a relationship (although weak) between the IT5 value and the interaural PTT difference for $6 \mathrm{kHz}$. Thus, the detection of a tumor by the standard IT5 measure appears to be dependent on the compromise of neural fibers higher in frequency than those assessed by the clinical PTA.

Figure 5 illustrates further evidence of this dependence of the IT5 on high-frequency fiber activity in two tumor patients of the current study. In both cases (fig. $5 \mathrm{a}$, b), responses in the left panel are from the non-tumor ear and responses in the right panel are from the ear with the tumor. In each panel, the top trace is the ABR to clicks alone at $60 \mathrm{~dB} \mathrm{nHL}$ and the lower traces are the derived-band responses representing activity initiated from octave-wide regions of the cochlea. The theoretical center frequencies of the derived bands are noted between the two panels of each figure. Figure 5 a shows the responses from a patient with a tumor in the left ear; the tumor dimensions noted in the chart were $5 \times 5 \times 3 \mathrm{~mm}$. The clinical PTA for the tumor ear was $47.5 \mathrm{~dB}$ and for the non-tumor right ear $32.5 \mathrm{~dB}$. The responses from the non-tumor ear in the left panel show that the wave $\mathrm{V}$ latency in the response to clicks alone appears to be dominated by activity from around the $5.7-\mathrm{kHz}$ region because the latencies are similar. In com- parison, the responses from the tumor ear displayed in the right panel show the latency of wave $\mathrm{V}$ in the response to clicks alone to be dominated by activity from around the $1.4-\mathrm{kHz}$ region because the latencies are similar and there is virtually no apparent synchronous activity in the three higher frequency derived-band responses. Thus, because the latency in the click alone response from the non-tumor ear is dominated by activity from the $5.7-\mathrm{kHz}$ high-frequency region while the latency in the click alone response from the tumor ear is dominated by activity from the $1.4-\mathrm{kHz}$ lower frequency region, a large abnormal IT5 (IT5 $=1.26 \mathrm{~ms}$ ) was measured. This case clearly demonstrates that because the tumor sufficiently compromised the high-frequency fibers that normally dominate the latency of wave $\mathrm{V}$ in the click alone response, the IT5 is abnormal and the tumor was detected. Also, note that the latency for the $1.4-\mathrm{kHz}$ derived-band response is less than $0.2 \mathrm{~ms}$ ( 8.23 vs. 8.08) longer than the same derived-band response for the non-tumor ear. Therefore, in this case, much of the large latency difference in the IT5 represents the cochlear difference between activity from the higher frequency and lower frequency regions, not neural delay caused by compression of the 8 th nerve.

Figure $5 \mathrm{~b}$ presents the click alone and derived-band $A B R$ responses from a patient with a tumor in the left ear; the tumor dimensions noted in the chart were $5 \times$ $9 \mathrm{~mm}$. The clinical PTA in the tumor ear was $11.25 \mathrm{~dB}$ and in the non-tumor ear $10.0 \mathrm{~dB}$. For both the non-tumor ear (left panel) and the tumor ear (right panel), the largest and most dominant response comes from the 5.7$\mathrm{kHz}$ region and the latencies of wave $\mathrm{V}$ in the $5.7-\mathrm{kHz}$ derived bands are identical. As a result, the latency of wave $\mathrm{V}$ in both click alone responses (top traces in both panels) is dominated by and equal to the latency of wave $\mathrm{V}$ in the $5.7-\mathrm{kHz}$ derived-band responses. Because the wave $\mathrm{V}$ latencies for the click alone responses are the same for both ears, the IT5 is normal and indicates no difference between the two ears (IT5 $=0$ ), and the tumor was missed. Note that the IT5 measure missed this tumor even though it is larger than the tumor detected by an abnormal IT5 in the previous case (fig. 5a). This case clearly demonstrates that without sufficient compromise of the high-frequency fibers that normally dominate the latency of wave $\mathrm{V}$ in the click alone response, the standard IT5 ABR measure will be normal and the tumor will be missed.

In addition, the two cases presented in figure 5 underscore the minor role that size has on detection of small tumors by the standard IT5 measure. The smaller tumor 


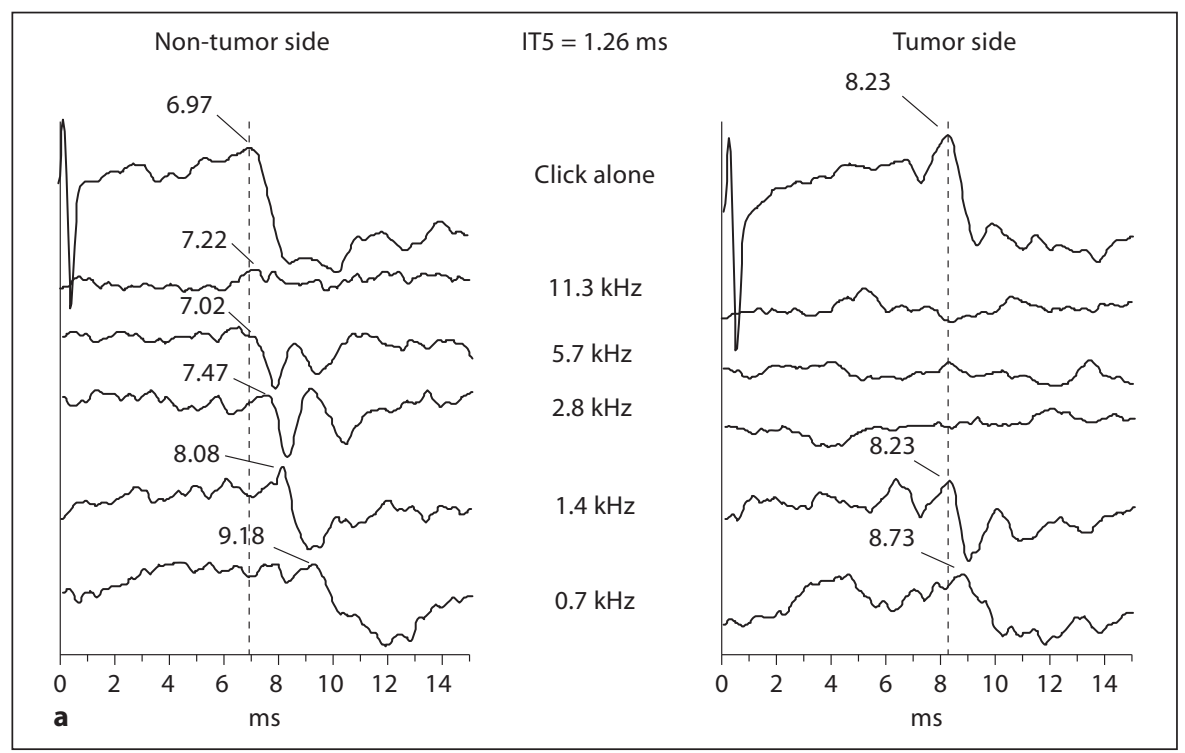

Fig. 5. Click alone and derived-band responses from tumor cases demonstrating the dependence of the IT5 measure on activity in the high-frequency regions of the cochlea. a No high-frequency activity on tumor side $=$ abnormal IT5. b Normal high-frequency activity on tumor side $=$ normal IT5. Also seen is a post-auricular muscle (PAM) response. See text for details.

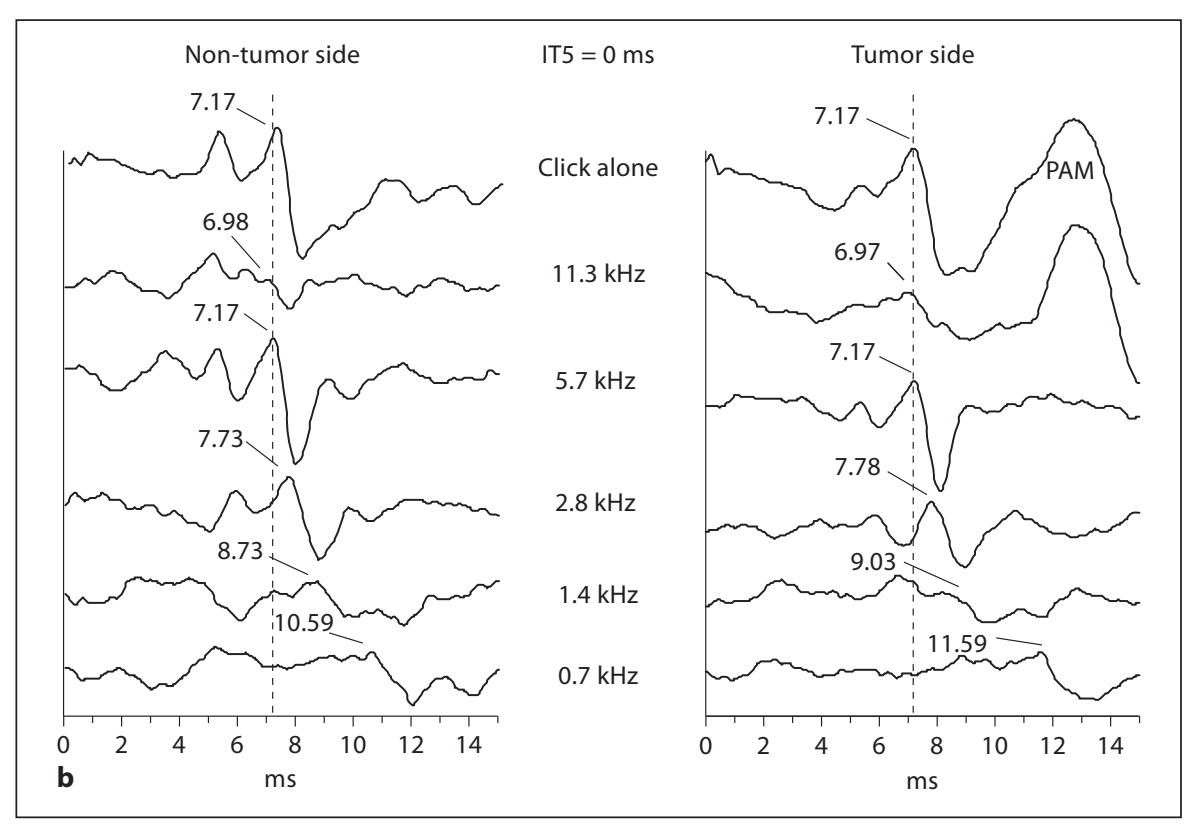

(fig. 5a) had a very abnormal IT5, suggesting that the tumor compromised either the high-frequency fibers directly by physical pressure or the vascular supply to the high-frequency cochlear hair cells or both. However, the IT5 was normal for the larger tumor (fig. 5b), so there appears to have been little direct or indirect compromise of the high-frequency neural or cochlear elements. Thus, for small tumors, size alone is less important than the tumor's location relative to neural or vascular components related to the high frequencies. Of course, with large tu- mors, the likelihood of compromising these neural or vascular elements is very high.

Detection of an acoustic tumor by the standard IT5 measure clearly requires sufficient compromise of the subset of high-frequency fibers that normally dominate the latency of wave V in standard click-evoked ABRs. Thus, the Stacked ABR was developed because it is an amplitude measure of synchronous activity involving essentially all fibers, not just the high-frequency ones. 


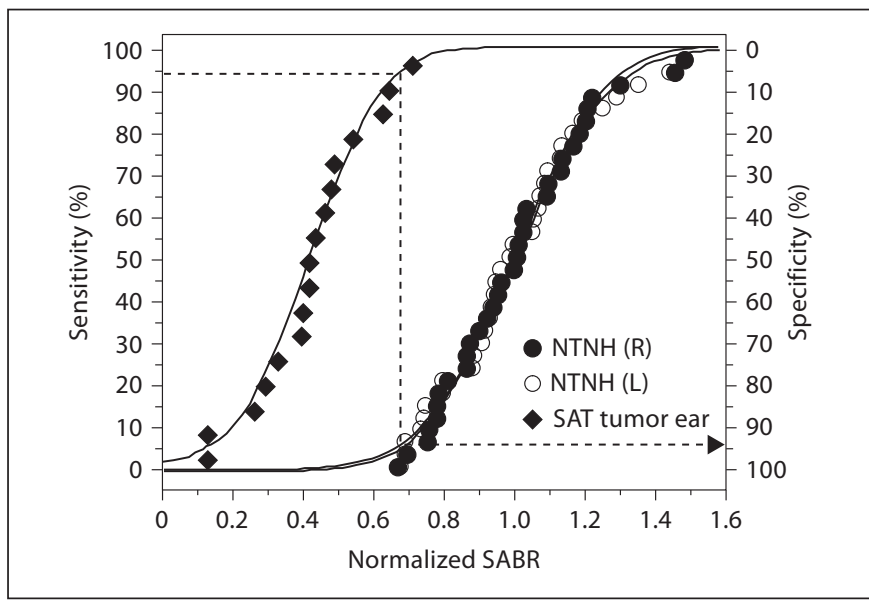

Fig. 6. Cumulative distributions of the normalized SABR amplitudes for the NTNH and SAT groups.

\section{SABR Data}

\section{Single Ear Comparisons}

The cumulative distributions of the normalized SABR amplitudes for both ears of the $39 \mathrm{NTNH}$ subjects and for the tumor ears of the 17 SAT cases are shown in figure 6 . The actual SABR amplitudes are normalized to the mean of the NTNH group for the appropriate gender (male or female) and ear (right or left). For example, if the tumor was in the right ear of a male patient, the SABR amplitude was divided by the mean for the right ear of males in the NTNH group. Similarly, if the tumor was in the left ear of a female patient, the SABR amplitude was divided by the mean for the left ear of females in the NTNH group, and so forth. Note that SABR values from earlier work [Don et al., 1997, 2005] were not included because those values were obtained using TDH-50 earphones, whereas ER-2 insert earphones were used in the present study. Based on the fitted cumulative distribution curve for the SAT population, the calculated $95 \%$ sensitivity results in a normalized SABR value of 0.688 . This normalized value, based on the fitted cumulative distribution curve for the NTNH population, yields a specificity of $93 \%$. These results are comparable to those of an earlier study [Don et al., 2005] in which $88 \%$ specificity was obtained for $95 \%$ sensitivity in a larger number (54) of small tumor cases. As emphasized in that study, the specificity is relative to SABRs from relatively young ( $<40$ years) NTNH adults. As mentioned earlier and discussed in previous work [Don et al., 2005], although age and hearing loss can significantly compro-

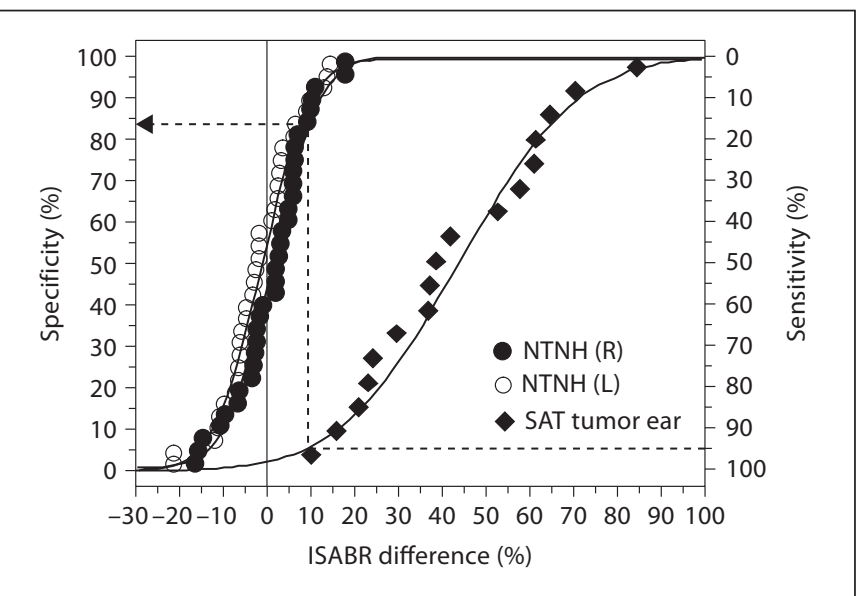

Fig. 7. Cumulative distributions of the percent interaural difference of the SABR amplitude for the NTNH and SAT groups. See text for details.

mise the specificity of the test, they will improve its sensitivity. Given the high sensitivity with single ear measures, the main value of an interaural comparison of the SABRs will be to improve specificity, especially for the patients discussed earlier who had either very large or very small normal SABR amplitudes.

\section{ISABR Comparisons}

The cumulative distributions of the percent difference in the ISABR amplitudes for the right and left ears of the $39 \mathrm{NTNH}$ subjects and for the tumor and non-tumor ears of the 17 SAT cases are shown in figure 7. For the SAT patients, the percent ISABR amplitude difference is calculated by subtracting the SABR amplitude for the tumor ear from the SABR amplitude of the non-tumor ear and dividing the result by the SABR amplitude of the nontumor ear. However, for the NTNH subjects, there is no tumor ear. Thus, the interaural difference is calculated and the cumulative distributions are plotted for two NTNH conditions: (1) NTNH (R) - when the right ear is considered the non-tumor ear and the left is considered the tumor ear, and (2) NTNH (L) - when the left ear is considered the non-tumor ear and the right is considered the tumor ear.

In a non-tumor subject population with normal hearing in both ears, the left and right ear SABR amplitudes should be essentially equal and the percent ISABR amplitude difference close to zero. Thus, as expected, the two NTNH cumulative curves in figure 7 overlap well and the 50th percentile is about zero percent for both curves. 


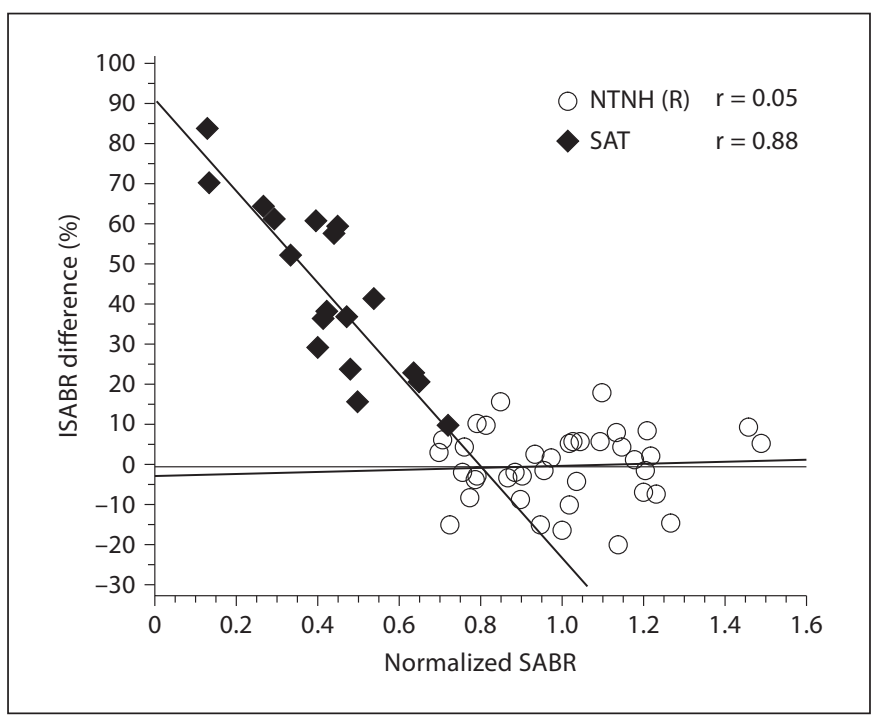

Fig. 8. The relationship between the normalized SABR amplitude (normalized to the right ear of the NTNH group) and the percent ISABR amplitude difference between ears for the NTNH group and the SAT group. A strong relationship exists only for the SAT group because of the effect of the tumor on the SABR.

With a calculated mean difference in the SABR amplitude of $0.002 \%(\mathrm{SD}=8.9 \%)$ for the NTNH $(\mathrm{R})$ data and $-0.768 \%$ (SD $=8.9 \%$ ) for the NTNH (L) data, the mean ISABR amplitude difference for the NTNH population is less than $1 \%$, demonstrating, as expected, essentially no difference between ears. However, for any given NTNH subject, the SABR amplitude may be a little larger on the right, while for others, it may be a little larger on the left. Thus, as seen in figure 7, the ISABR amplitude differences for the NTNH group fall both above and below $0 \%$. For the ISABR amplitude difference in an NTNH subject to fall below $0 \%$, the SABR amplitude from the designated 'tumor' ear must be greater than that from the designated 'non-tumor' ear. Note that only the NTNH group has percent ISABR amplitude differences less than $0 \%$.

Figure 7 also shows that, as expected, the percent ISABR amplitude differences are greater for the SAT patients than for the NTNH subjects. All of the 17 tumor ears had percent ISABR amplitude differences greater than $0 \%$. Thus, in the SAT group, the SABR amplitude from the tumor ear was always smaller than that from the non-tumor ear. The calculated mean (approximately the $50 \%$ point in the cumulative distribution) ISABR difference for the SAT patients is $43 \%$ ( $\mathrm{SD}=21.3 \%$ ). Therefore, on average, a small tumor will reduce the SABR amplitude by $43 \%$ relative to the non-tumor side.

Interaural Stacked Auditory Brainstem Responses
Using these data to assess the sensitivity and specificity of the ISABR measure for this SAT population, we calculated that for a sensitivity of $95 \%$, the interaural difference is about $8 \%$ and this yields a specificity of about $83 \%$. While this specificity is lower than for the single ear value shown in figure 6 , it is fairly comparable to the sensitivity (95\%) and specificity (88\%) for the single ear SABRs published earlier by Don et al. [2005]. Moreover, it should be remembered that this interaural comparison may help when testing a non-tumor patient whose normal SABRs are at the low end of the NTNH distribution or a tumor patient whose SABRs are normally at the high end. For example, if the SABR criterion for the single ear analysis was set for $80 \%$ specificity, then the $20 \%$ of nontumor subjects with SABR amplitudes below the criterion would have false-positive results and be mistakenly diagnosed as having a tumor. However, as mentioned above and shown in figure 7 , the mean ISABR difference for the NTNH group is close to 0, while for the SAT patients, it is $43 \%$. Thus, the interaural difference in SABR amplitudes is much greater for true tumor patients than for non-tumor subjects. As a result, it is probable that in the NTNH subjects with SABR amplitudes in the lower 20\% of the distribution, the interaural measure will reveal little difference. Thus, even though the single ear SABR analysis based on a criterion of $80 \%$ specificity mistakenly suggests that these NTNH subjects have a tumor, the ISABR measure leads to the correct diagnosis of no tumor. A more concrete example of this use of the ISABR amplitude measure can be illustrated using non-tumor subjects (fig. 6) having normalized SABR amplitudes that are at the low end of the distribution. In figure 6 , the two non-tumor subjects with the smallest amplitudes have normalized SABR values that are 0.68 and 0.69 . These values overlap the high end of the tumor distribution for the SAT patients and are in the range that would be positive for a tumor for a sensitivity criterion set at $95 \%$. Thus, based on the single ear distribution of normalized SABR amplitudes, these two subjects would be incorrectly diagnosed as having tumors (i.e. false-positive cases) and the specificity of the test would be compromised. However, the percent ISABR amplitude differences for these two subjects are 1.7 and $3.2 \%$. As can be seen in figure 7 , these interaural differences fall well within the NTNH percent ISABR difference distribution. Thus, using the ISABR measure yields the correct diagnosis of no tumor. This is an example of how using the percent ISABR difference as an additional measure can improve specificity by reducing the number of false positives based on single ear SABR analyses alone.

Audiol Neurotol 2012;17:54-68 
The fact that one measure (the single ear normalized SABR amplitude) can be interpreted as being positive for a tumor while a second measure (the percent ISABR difference) is negative for a tumor, suggests that these two measures are independent. Figure 8 shows the relationship between the normalized SABR amplitude and the percent ISABR difference for the NTNH subjects and the SAT patients. For the NTNH group, only NTNH (R) data are plotted. Thus, the normalized SABR amplitude values were determined by dividing each NTNH subject's right ear SABR amplitude by the mean for the right ear of the appropriate gender (male or female) in the NTNH group (as described earlier for figure 6), and the percent ISABR difference for each NTNH subject was determined by subtracting the SABR amplitude for the left ear from the SABR amplitude of the right ear and dividing the result by the SABR amplitude of the right ear (as described earlier for figure 7).

It can be seen from figure 8 that for the right ear of the NTNH group, there is no relationship as the correlation $\mathrm{r}$-value is $0.05(\mathrm{p}=0.72)$. However, when using the NTNH (L) data, a larger $r$-value of $0.32(p=0.05)$ was obtained suggesting a weak correlation. For the SAT group, the actual SABR amplitudes are normalized to the mean of the NTNH group for the appropriate gender (male or female) and ear (right or left) as described earlier for figure 6. It can be seen that for the SAT patients, there is a definite relationship with an r-value of $0.88(\mathrm{p}>0.0001)$. That is, small normalized SABR values and large interaural differences in the SABR values are due to the presence of a tumor. Thus, for the SAT group, the smaller the normalized SABR amplitude, the greater the SABR amplitude difference between ears. Whereas for the NTNH group, there is no tumor and the values are, at best, weakly correlated.

\section{Effect of Hearing Loss on SABR and ISABR Measures}

An important consideration in ISABR measures, as well as the single ear SABR, is the effect of hearing loss. In general, hearing loss not due to a tumor, such as presbycusis or a noise-induced hearing loss, will reduce the amount of synchronous neural output and the SABR amplitude. In the tumor ear, hearing loss will improve the sensitivity of the SABR measures because both the tumor and the hearing loss will reduce the SABR amplitude on the tumor side and increase the ISABR difference. However, if in a tumor patient there is hearing loss on the nontumor side that is significantly greater than the hearing loss on the tumor side, this will compromise the sensitivity of the ISABR measure. This is because the SABR amplitude reduction in the tumor ear due to the tumor may be matched by a reduction in amplitude in the non-tumor ear due to the greater hearing loss, resulting in a small interaural difference (i.e. small ISABR value). The issue of how to compensate for hearing loss will be addressed more fully in a subsequent paper.

However, some insight as to how we can compensate for the effect of hearing loss is provided in figure 9 in which we plot for the 17 tumor cases the percent ISABR amplitude difference as a function of the difference in the clinical PTA for the two ears. An important assumption in plotting these data is that for a given individual, equal amounts of hearing loss in each ear would produce a comparable reduction of the SABR amplitude. By plotting these data in this way, we can assess the effect of the tumor and estimate the effect of hearing loss. Such a plot also removes the influence of the variability of the absolute values across subjects because the percent ISABR differences are relative measures. Furthermore, by using interaural comparisons, the effect of age is also removed because it must be assumed that the two ears of each individual are affected by age in the same way. In figure 9 , there is a strong simple linear relationship $(r=0.88, p<$ 0.0001 ) between the percent ISABR amplitude difference and the difference in the clinical PTA. The point of intersection with the ordinate at $0 \mathrm{~dB}$ PTA difference is about $20 \%$. This suggests that the effect of the tumor's presence alone is to reduce, on average, the SABR amplitude by $20 \%$. The slope describes the rate of reduction as a function of the difference in hearing loss, and in this case, it is about $1.35 \%$ per $\mathrm{dB}$ clinical PTA difference. Thus, based on these data, the predicted percent ISABR difference due to the presence of a tumor is the combination of $20 \%+1.35 \%$ per $\mathrm{dB}$ clinical PTA difference.

We recognize that figure 9 is quite simplistic and describes an average and linear effect of the amount of decrement caused by (1) direct impingement from the tumor and (2) any resultant interaural difference in cochlear hearing loss as described broadly and incompletely by the audiometric thresholds. We also recognize that it is likely that this linear relationship will not be an adequate description as the hearing loss at all frequencies in either ear approaches $60 \mathrm{~dB}$ HL given that our stimulus level is only $60 \mathrm{~dB}$ nHL. However, it should be recalled that patients with small tumors often have minimal hearing loss. Thus, the data in figure 9 do give a general guideline for much of the range of interaural hearing loss differences encountered in patients with small tumors. For example, shown in figure 9 is a regression line (dashed) with the same slope ( $1.35 \%$ per dB clinical PTA difference) but shifted by $20 \%$ to remove the estimated effect of the tu- 


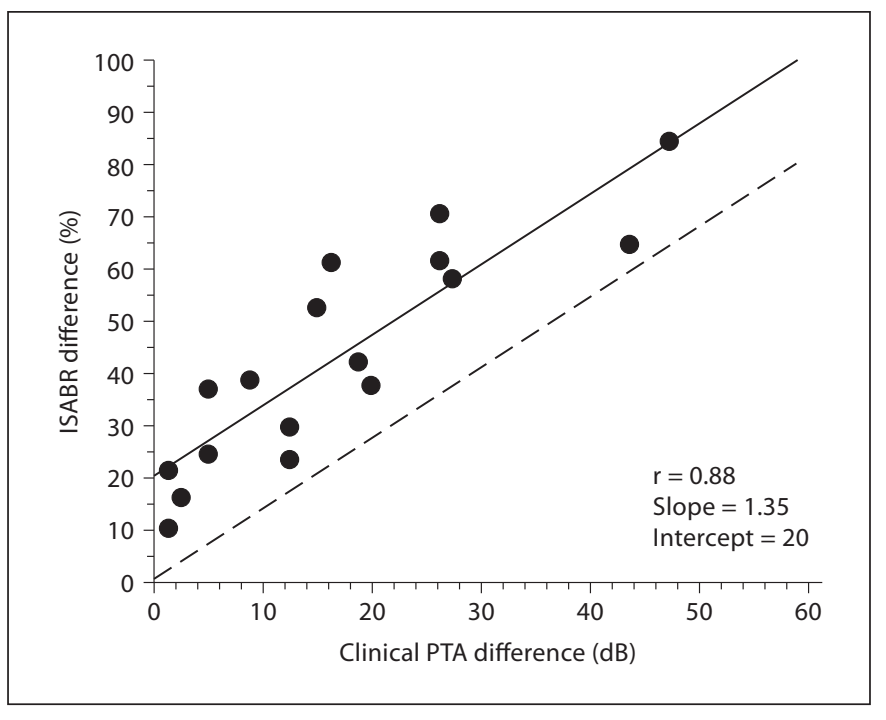

Fig. 9. Percent ISABR amplitude difference plotted as a function of the interaural clinical PTA difference. Modified with permission from Don [2007].

mor. A simplistic criterion is to be suspicious of any patient whose ISABR difference falls above this line because the difference is greater than predicted by the hearing loss difference alone. Note that the data from all 17 tumor cases lie above this line. However, as we indicated earlier, the main value for hearing loss compensation is to improve the specificity, that is, to reduce the number of false positives that occur because of cochlear hearing loss. A careful assessment of the specificity of the ISABR measure requires a well-controlled study of patients with varying amounts of hearing loss unrelated to otologic diseases such as acoustic tumors that could cause additional compromise of the SABR amplitude. We plan to report on such a study in a future paper.

\section{Discussion}

As noted earlier, for the test populations in this study, the calculated sensitivity and specificity for the standard IT5 criterion of $0.2 \mathrm{~ms}$ recommended by Selters and Brackmann [1977] are 78\% and 97-98\%, respectively. The specificity is high, but it is for an IT5 criterion value that missed $22 \%$ of the small tumors. If instead of the $0.2-\mathrm{ms}$ criterion value recommended by Selters and Brackmann [1977], we use a criterion of 95\% sensitivity, the sensitivity reported for the SABR and ISABR measures, the calculated specificity of the IT5 measure drops to less than
$1 \%$ (see description of figure 3). Previously [Don et al., 1997, 2005; Don and Kwong, 2009], we hypothesized that the standard IT5 latency measure, as well as any other ABR measure based on latency measures, failed to detect some small tumors because the small tumor did not compromise the high-frequency fibers sufficiently. We present some data consistent with this hypothesis as we show evidence in figure $4 \mathrm{~b}$ suggesting that the standard IT5 tended to detect small tumors in patients whose $6-\mathrm{kHz}$ PTT was greater in the tumor ear. Interaural differences for other PTTs did not correlate with tumor detection by the IT5 measure. In addition, the two cases in figure 5 suggest that small tumor detection by the IT5 measure does not depend on the tumor's size per se, but on its location. Clearly, if the tumor does not sufficiently compromise either the higher frequency fibers directly or the vascular supply to high-frequency sensory elements in the cochlea or both, the IT5 will be normal and the tumor will be missed.

Thus, the failure of the IT5 to detect small tumors is not due to inappropriate use of a correction factor based on the $4-\mathrm{kHz}$ threshold, but due to insufficient compromise of the high-frequency fibers that dominate the latency of wave V. However, note that correcting for highfrequency (i.e. $4-\mathrm{kHz}$ ) hearing loss does not increase the sensitivity of the IT5. In fact, it will tend to reduce its sensitivity because, similar to the effect of general hearing loss on the ISABR discussed earlier, high-frequency hearing loss in a tumor ear actually improves the sensitivity of the IT5 because both the tumor and the high-frequency hearing loss increase the latency of wave $\mathrm{V}$ on the tumor side and increase the IT5. Correcting for high-frequency hearing loss will, at best, increase the specificity of the IT5, that is, increase the correct identification of non-tumor subjects because the correction factors will offset the increased latency of wave V caused by the highfrequency hearing loss and decrease the IT5. We stress in this discussion that high-frequency hearing loss leads to a prolonged wave $\mathrm{V}$ latency to clicks, but general hearing loss can lead to shorter wave latencies [Don et al., 1998]. This further emphasizes the difficulties in using wave $\mathrm{V}$ latency measures when hearing loss is also involved.

We have proposed the use of an ISABR amplitude measure to help screen for small unilateral acoustic tumors. In a previous paper [Don et al., 2005], we demonstrated that the SABR amplitude is significantly reduced in patients with small $(\leq 1 \mathrm{~cm})$ tumors. In that study, for the SABR measure, the criterion amplitude providing $95 \%$ sensitivity resulted in $88 \%$ specificity; whereas for the IT5 measure, the criterion latency difference providing $95 \%$ sensi- 
tivity resulted in a specificity close to $0 \%$. Because the standard IT5 ABR measure will detect nearly all large and medium-sized tumors (and should, therefore, be the first test measure), the SABR was aimed at individuals with small tumors that are missed by the standard IT5 measure. In an earlier work [Don et al., 2005] and again in this study (fig. 6), the normalized SABR amplitudes indicate that the range of amplitudes in the sample NTNH population was large, with the highest observed amplitude being a little more than twice that of the lowest amplitude observed. Such a large range can affect both the sensitivity and the specificity of the SABR measure. For example, as discussed earlier, if individuals who normally have very large SABR amplitudes (i.e. SABR amplitudes at the high end of the distribution) develop a small tumor, the reduction in the SABR amplitude may not be sufficient to reach the abnormal range for the single ear distributions. Thus, the tumor would be missed in these cases and the test's sensitivity would be compromised. On the other hand, non-tumor individuals with SABRs at the low end of the distribution may fall below the criterion for the single ear distribution, resulting in a diagnosis of an acoustic tumor. This would be a false positive compromising the specificity of the single ear SABR measure.

To improve the use of SABR measures, we developed an ISABR amplitude measure that is the percent difference calculated by subtracting the SABR amplitude for the suspected tumor ear from the SABR amplitude of the non-tumor ear and dividing the result by the SABR amplitude of the non-tumor ear. The justification for this measure is the observation that the mean interaural difference in the SABR amplitudes in NTNH subjects is less than $1 \%$ (see fig. 7). Figure 7 also shows that, as expected, the percent ISABR amplitude differences are much greater for the SAT patients than for the NTNH subjects. Moreover, note that all of the differences for the SAT group are positive, that is, the amplitude of the non-tumor ear is always larger in this group. The calculated mean percent ISABR difference for the SAT patients is $43 \%$. Note, that this $43 \%$ ISABR mean difference is slightly less than the mean reduction of $50 \%$ observed in our recent study [Don et al., 2005] comparing SABR amplitudes between SAT patients and NTNH subjects. This is to be expected since the SAT patients in the current study are older than the NTNH subjects in the 2005 study and most had some hearing loss in the non-tumor ear. In addition, note that because the ISABR is a relative withinsubject measure, it will not be affected by the large intersubject variability in absolute SABR amplitude. The results (fig. 7) from this study suggest that $95 \%$ sensitivity and $83 \%$ specificity can be achieved by using an ISABR difference criterion of $8 \%$.

It should be noted that the sensitivity and specificity of the ISABR measure differ little from those for the single ear SABR. This raises the question as to whether we can truly claim that using an ISABR measure will improve the sensitivity and specificity of SABR measures. Perhaps the main way that the ISABR measure 'improves' the specificity and sensitivity of SABRs is by restoring the diagnostic value of the SABR in conditions that would be detrimental to the sensitivity and specificity of the single ear distributions. As discussed earlier, these detrimental conditions occur in tumor patients with normally large SABR amplitudes and non-tumor patients with normally low SABR amplitudes. In these situations, the single ear comparisons would fail whereas the use of the ISABR measure would have a high probability of providing the correct diagnosis. It was shown earlier how the ISABR could improve specificity by clarifying the interpretation of the low SABR amplitudes of a couple of NTNH subjects. Their low SABR amplitudes could have been interpreted as positive signs of a tumor. However, the percent ISABR amplitude differences were well within the NTNH range. In these cases, the ISABR measure provided invaluable additional information that led to the correct diagnosis.

Note that the single ear distributions of the SABR will have to be used when the ISABR measure is either unobtainable due to lack of testing time or inappropriate because of neurofibromatosis type II and the possibility of bilateral tumors. In addition, a caution to note is that the single ear absolute amplitude norms established earlier [Don et al., 2005] and used in the commercially developed Natus/Bio-logic Stacked ABR system, are specific to the data collection techniques and analysis methods used in that study. Variability across clinics involving environmental factors (e.g. sound room or no sound room), methods for recording the ABRs (e.g. electrode application and location), methods affecting stimulation (e.g. ear tip insertion depth), and analyses (e.g. peak selection), as well as minor hardware/software differences, may affect the SABR amplitudes. This variability and other clinical issues that can adversely affect and even invalidate test outcomes will be addressed in detail in a forthcoming paper. It is strongly recommended that each clinical facility establish their own normative data for their specific recording instrumentation and conditions. If clinicians wish to use the criteria specified by the software of the Natus/Bio-logic commercial system and not establish their own norms, then strict adherence to the data collection techniques and analysis methods used in the Don et 
al. [2005] study is necessary. That is, it is imperative that the recording and stimulating conditions, as well as the analyses, be similar to those of Don et al. [2005] to avoid compromise of the SABR's sensitivity and specificity. We caution that even strict adherence to the data collection techniques and analysis methods published by Don et al. [2005] may not be sufficient if the minor hardware differences between the Don et al. [2005] laboratory system and the commercial system produced by Natus/Bio-logic have significant impact on the SABR absolute amplitude measures. However, by using the ISABR measure, these variables have less effect as long as the conditions are the same for each ear tested. Thus, an additional advantage of the ISABR measure is its greater immunity to variables that affect absolute amplitude values.

Finally, as noted earlier, hearing loss reduces synchronous neural output and thus, the SABR amplitude. If there is hearing loss in a tumor ear, the sensitivity of the SABR measure improves because both the tumor and the hearing loss reduce the SABR amplitude. But if there is a significantly greater hearing loss in the non-tumor ear of a tumor patient, the reduction in the SABR amplitude due to the tumor may be matched by the reduction due to the hearing loss in the non-tumor ear. As a result, not only will the specificity of the SABR measure be adversely af fected due to the significantly reduced SABR amplitude in the non-tumor ear, but the interaural amplitude difference will be reduced and the sensitivity of the ISABR measure will be compromised. A method of compensating for the hearing loss would be valuable in these cases. As an initial effort to develop one, we demonstrated that in the current test population of small tumors, each $\mathrm{dB}$ difference in the interaural clinical PTA reduced the SABR amplitude by an additional $1.35 \%$. Until a full study of the effect of hearing loss can be performed, we suggest that $1.35 \%$ per $\mathrm{dB}$ be used as a general guideline in compensating for the effect of hearing loss in small tumor cases.

However, we must be cautious in attempting to improve specificity by compensating for hearing loss because this will reduce sensitivity and the original problem with using ABRs in small tumor cases was the lack of sensitivity. While we have proposed a tentative approach to compensating for the effects of hearing loss, future work is needed to independently validate the approach and the compensation values derived in the population of this study.

Clinically, the IT5, SABR, and ISABR measures can be used together to maximize efficient detection of small unilateral acoustic tumors. The first test should be the standard IT5 [Don and Kwong, 2009] because, as noted before, it is a relatively fast and simple test that detects nearly all medium and large tumors, as well as $50-70 \%$ of small tumors [Don, 2002]. If the IT5 is normal, then the SABR measure should be performed [Don and Kwong, 2009] because, as discussed extensively, the SABR test targets small tumors with high sensitivity and specificity. If the results of the SABR measure are questionable in cases with conditions detrimental to its sensitivity and specificity, for example, in tumor patients with normally large SABR amplitudes or non-tumor patients with normally low SABR amplitudes, then the percent ISABR amplitude difference should be calculated because, as demonstrated earlier, the ISABR measure can provide invaluable additional diagnostic information. By using the IT5 as the first test, the SABR amplitude measure as the second test, and the percent ISABR amplitude difference measure as the third test, the clinician has a progressive screening protocol that minimizes the chances of missing a small $(\leq 1 \mathrm{~cm})$ unilateral acoustic tumor.

\section{Conclusion}

We have introduced an ISABR amplitude difference measure to improve the sensitivity and specificity of the SABR amplitude measure to detect small unilateral acoustic tumors. It has been shown to have both high sensitivity and fairly high specificity. Its main advantages are two-fold. First, it is somewhat immune to variables that affect the absolute SABR amplitudes because it is a relative measure. Second, it is better at assessing tumor patients with very large and non-tumor patients with very small absolute SABR amplitudes. We believe that the ISABR is a useful addition to ABR measures aimed at detecting the presence of SAT.

\section{Acknowledgements}

We thank Michael Waring, $\mathrm{PhD}$ for his technical support. We also thank the physicians and members of the House Clinic Staff for their help in recruiting patients for this study. This project was supported by a grant (number R01 DC003592) from NIDCD at NIH to M.D. (P.I.). Its contents are solely the responsibility of the authors and do not necessarily represent the official views of the NIDCD or NIH. 


\section{References}

Carhart R, Jerger JF: Preferred method for clinical determination of pure-tone thresholds. J Speech Hear Disord 1959;24:330-345.

-Don M: Auditory brainstem response testing in acoustic neuroma diagnosis. Curr Opin Otolaryngol Head Neck Surg 2002;10:376-381.

Don M: Hearing Loss can muddy the waters of otologic disease detection; in Dau T, Buchholz JM, Harte JM, Christiansen TU (eds): Auditory Signal Processing in Hearing-Impaired Listeners, International Symposium on Audiological and Auditory Research (ISAAR 2007). Helsingør, Denmark, 2007.

Don M, Eggermont JJ: Analysis of the clickevoked brainstem potentials in man using high-pass noise masking. J Acoust Soc Am 1978;63:1084-1092.

Don M, Elberling C: Evaluating residual background noise in human auditory brainstem responses. J Acoust Soc Am 1994;96:27462757.

Don M, Elberling C: Use of quantitative measures of ABR peak amplitude and residual background noise in the decision to stop averaging. J Acoust Soc Am 1996;99:491-499.
Don M, Kwong B: ABR: Differential diagnosis; in Jack Katz (ed): Handbook of Clinical Audiology, Sixth Edition. Pennsylvania, Lippincott Williams \& Wilkins Publishing, Media, 2009, pp 274-297.

Don M, Kwong B, Tanaka C, Brackmann D, Nelson R: The Stacked ABR: A sensitive and specific screening tool for detecting small acoustic tumors. Audiol Neurotol 2005; 10 274-290.

Don M, Masuda A, Nelson RA, Brackmann DE: Successful detection of small acoustic tumors using the stacked derived band ABR method. Am J Otol 1997; 18:608-621.

-Don M, Ponton CW, Eggermont JJ, Masuda A: Auditory brainstem response (ABR) peak amplitude variability reflects individual differences in cochlear response times. J Acoust Soc Am 1994;96:3476-3491.

Don M, Ponton CW, Eggermont JJ, Kwong B: The effects of sensory hearing loss on cochlear filter times estimated from ABR latencies. J Acoust Soc Am 1998;104:2280-2289.
Elberling C, Don M: Quality estimation of averaged auditory brainstem responses. Scand Audiol 1984;13:187-197.

Elberling C, Wahlgreen D: Estimation of auditory brainstem responses, $\mathrm{ABR}$, by means of Bayesian inference. Scand Audiol 1985;14: 89-96.

Musiek FE, Shinn JB, Jirsa RE: The auditory brainstem response in auditory nerve and brainstem dysfunction; in Burkard RF, Eggermont JJ, Don M (eds): Auditory Evoked Potentials: Basic Principles and Clinical Application. Philadelphia, Lippincott Williams \& Wilkins, 2007, pp 291-312.

Rosenhamer HJ, Lindström B, Lundborg T: On the use of click-evoked electric brainstem responses in audiological diagnosis: III. Latencies in cochlear hearing loss. Scand Audiol 1981;10:3-11.

- Selters WA, Brackmann DE: Acoustic tumor detection with brain stem electric response audiometry. Arch Otolaryngol 1977;103:181187. 